\title{
Evaluación del déficit de bosques de ribera en Tucumán
}

\author{
Edgardo J. I. Pero ${ }^{1, \Phi}$; Elvira Casagranda ${ }^{2}$; Luciana Cristobal ${ }^{1}$; Aldana Wottitz ${ }^{3}$ \& \\ NÉSTOR I. GASPARRI ${ }^{2}$ \\ ${ }^{1}$ Instituto de Biodiversidad Neotropical. Consejo Nacional de Investigaciones Científicas y Técnicas (CONICET) - Universidad \\ Nacional de Tucumán (UNT). Facultad de Ciencias Naturales e IML. San Miguel de Tucumán, Argentina. ${ }^{2}$ Instituto de \\ Ecología Regional. CONICET - UNT. Yerba Buena, Tucumán, Argentina. ${ }^{3}$ Facultad de Ciencias Forestales. Universidad \\ Nacional de Santiago del Estero. Santiago del Estero, Argentina.
}

\begin{abstract}
Resumen. Los bosques de ribera se encuentran entre los ecosistemas más vulnerables, y para mantener los servicios que brindan se necesita su restauración ecológica. El objetivo principal de nuestro trabajo fue generar un mapa de zonas ribereñas a fin de identificar bosques, áreas transformadas y oportunidades para restaurar estos ecosistemas en Tucumán, Argentina. Comparamos diferentes anchos de zonas de amortiguación (ZdA): según requisitos legales $(60 \mathrm{~m})$ y sugerencias científico-técnicas (100 y $250 \mathrm{~m})$. Para explorar la superficie de unidades de producción agrícola (UPA, lotes catastrales) ubicadas en las ZdA analizamos diferentes regiones agroecológicas y porcentajes de superficie ocupada en las UPA. La cobertura del suelo se cartografió a partir de una clasificación supervisada de imágenes Sentinel en Google Earth Engine. Nuestros resultados revelaron que para alcanzar a cubrir los 60 y $250 \mathrm{~m}$ de ZdA se necesitaría restaurar entre 40 y $60 \%$ de las zonas ribereñas, respectivamente, en su mayoría bajo agricultura. Si se considera una ZdA de $60 \mathrm{~m}$, las riberas se encuentran ocupadas por usos agrícolas y urbanos en $23.5 \%$ (1959 ha) en el pedemonte, 50.9\% (7784 ha) en la llanura húmeda y $36.8 \%$ (3113 ha) en la llanura seca. Nuestros resultados sugieren que se deben realizar diferentes esfuerzos para restaurar de manera eficiente las distintas regiones agroecológicas. La restauración necesaria para cubrir el requisito legal podría ser una meta alcanzable considerando objetivos globales y regionales. La mayoría de las UPA incluyeron menos de $20 \%$ de su superficie en una zona ribereña. Sin embargo, se recomienda incorporar abordajes socio-ecológicos en los proyectos de restauración, a fin de conocer las visiones de los productores, proponer compensaciones y evitar restricciones que afecten la producción agrícola local. El mapeo realizado será una herramienta valiosa para implementar proyectos de restauración en un área prioritaria de la Argentina.
\end{abstract}

[Palabras clave: vegetación de ribera, restauración ecológica, agricultura, gestión ambiental, zona de amortiguación]

\begin{abstract}
Aвstract. Evaluation of the riparian forest deficit in Tucumán. Riparian forests are among the most vulnerable ecosystems. Ecological restoration is needed to maintain the ecosystem services they provide. The main goal of the present study was to generate a map of riparian zones identifying forest and transformed areas as a base for their restoration in Tucumán, Argentina. We compared different buffers width: one according to legal requirement $(60 \mathrm{~m})$ and two scientific-technical suggestions $(100$ and $250 \mathrm{~m})$. To explore the occupation of the agronomic production units (cadastral units) by the buffers, we analyzed riparian land cover among different agro-ecological regions and the percentage of production units area affected. Land cover maps were performed through a supervised classification of Sentinel in Google Earth Engine. Our results revealed that between a 40 and 60 percent of the riparian zones, mainly covered by agriculture, need to be restored to reach the 60 and $250 \mathrm{~m}$ of buffer strip respectively. Considering the $60 \mathrm{~m}$ buffer, riparian zones were covered by agricultural and urban uses in a $23.5 \%$ (1959 ha) in the foothills, 50.9\% (7784 ha) in the wet plains, and $36.8 \%$ (3113 ha) in the dry plains. Therefore, different efforts must be made for an efficient and effective restoration. The amount of area needed to restore the legal requirement could be an attainable objective considering the global and regional restoration goals. In addition, majority of the production units included less than $20 \%$ of their surface in a riparian zone. However, we suggest including socio-ecological approaches for restoration projects to know social actors perceptions and avoid major restriction for agronomic production. The cartography performed will be a valuable tool for the implementation of restoration projects for a priority area in Argentina.
\end{abstract}

[Keywords: riparian vegetation, ecological restoration, land uses, agriculture, environmental management, buffer strip]

Editor asociado: Pablo Villagra
Recibido: 13 de Marzo de 2020

Aceptado: 19 de Agosto de 2020 


\section{INTRODUCCIÓN}

A los bosques de ribera se los considera una interfaz entre ecosistemas terrestres y acuáticos. Se encuentran entre los ambientes más amenazados tanto por la deforestación como por el cambio climático (Capon et al. 2013). De acuerdo con Naiman et al. (2005), los bosques de ribera se definen como la vegetación leñosa adyacente a ríos y arroyos. Estos bosques se extienden lateralmente desde el canal activo hasta zonas más elevadas, incluyendo áreas de inundación y las terrazas inmediatas. Esta interfaz tierra-agua es clave debido a la variedad de servicios ecosistémicos (SE) que provee y por las funciones que cumple. Por ejemplo, aportan materia orgánica e inorgánica a los ríos, moderan la temperatura del agua, regulan la entrada de nutrientes y contaminantes, mejoran la calidad ecológica del agua y estabilizan los márgenes de ribera. Además, brindan otros SE, tales como almacenar carbono, regular el ciclado de nutrientes y proveer hábitat y conectividad para la fauna, entre otros (Pokrovsky 2016; Riis et al. 2020).

Actualmente, se reconoce que la restauración de bosques es una prioridad ambiental global (Suding et al. 2015); fue incluida como parte de los objetivos de desarrollo sustentable (ODS) en la Agenda de las Naciones Unidas para el año 2030 (ONU 2015). Alrededor del planeta, muchos paisajes ribereños fueron modificados para aumentar la producción agrícola y establecer asentamientos humanos (Posthumus et al. 2010), lo cual afecta el funcionamiento de estos ecosistemas y su capacidad para proveer SE (Naiman et al. 2005). Esta situación amerita atención y se puede mejorar a través de la restauración ecológica (RE) (Barral et al. 2015; LozanoBáez et al. 2019). Sin embargo, los criterios para definir y delimitar zonas ribereñas y dimensiones óptimas de amortiguación o buffers donde llevar a cabo las actividades de RE varían según los parámetros a considerar (i.e., hidrológicos, florísticos, geomorfológicos, legales) (Tiwari et al. 2016). Las variaciones ecorregionales de la estructura y la composición de los bosques de ribera sugieren tener en cuenta una perspectiva del paisaje para la toma de decisiones de gestión ambiental (Pero and Quiroga 2019). Además, diversos estudios revelaron que una planificación previa y a escala del paisaje de los programas de restauración maximiza los resultados positivos del proceso (Aronson et al. 2017; Strassburg et al. 2019).
En Latinoamérica se evidencia un interés creciente en la restauración de ecosistemas boscosos (Meli et al. 2019; Schweizer et al. 2019). En la Argentina se están llevando a cabo diversos proyectos de RE, especialmente luego de la sanción de la Ley de Presupuestos Mínimos de Protección Ambiental de los Bosques Nativos (Ley 26331) y del establecimiento del Plan Nacional de Restauración de Bosques (Pérez et al. 2018; de Paz et al. 2019; Kutschker et al. 2020). En este contexto, aplicar técnicas modernas para generar mapas detallados que permitan localizar y cuantificar áreas con déficit de bosque es esencial para planificar proyectos de RE más eficientes y eficaces (Macfarlane et al. 2016). Este tipo de insumo científicotécnico puede favorecer la articulación entre enfoques impulsados 'por políticas top-down (de arriba hacia abajo) que establecen objetivos nacionales y regionales, con objetivos locales basados 'en el contexto socio-político, y necesidades locales bottom-up (de abajo hacia arriba) (Meli et al. 2019).

En las zonas subtropicales de la Argentina, los bosques pedemontanos y de llanura están entre los ecosistemas más modificados y amenazados a causa de los cambios de uso del suelo que tuvieron lugar en las últimas décadas (Gasparri et al. 2016). En el año 1986, los bosques ocupaban $62 \%$ de las riberas de la llanura de Tucumán, a una distancia de $500 \mathrm{~m}$ desde los ríos. En el año 2010 pasaron a ocupar $40 \%$ de estas zonas, luego de que $\sim 24000$ ha de estos bosques (principalmente bosque seco) fueran convertidas a agricultura (Díaz Gómez and Gaspari 2017). En particular, la llanura tucumana es una región con una historia larga de conversiones del uso del suelo. En la actualidad, más de un millón de personas viven en esta área donde se concentra la principal producción agrícola de la provincia, que representa el núcleo de la economía regional. Sin embargo, la región es afectada regularmente por inundaciones que impactan sobre la población, la infraestructura y las actividades productivas (Díaz 2018). Esta situación plantea preocupaciones sobre la sostenibilidad y la integridad ambiental de la región para enfrentar futuros escenarios de cambio climático y de usos del suelo. En la comunidad local, incluida la sociedad civil, los/las académicos/as y los/las tomadores de decisiones, existe un acuerdo común de que la pérdida de bosque de ribera es uno de los problemas clave que promueven la inestabilidad del curso de los ríos y las inundaciones (Comisión de Emergencia 
para El Tratamiento de la problemática de inundaciones en el sur de la provincia de Tucumán, Este de Catamarca y Río Hondo. 2017. Informe Técnico (tinyurl.com/yyce8bec). A pesar de este contexto, hasta este momento no existen estimaciones de la superficie de bosque de ribera y usos del suelo en el área.

En el presente estudio hemos utilizado técnicas de teledetección y sistemas de información geográfica para contestar las siguientes preguntas sobre el pedemonte y la llanura de la provincia de Tucumán, en el noroeste de la Argentina: 1) ¿Cuál es la superficie de bosque de ribera considerando diferentes medidas de amortiguación definidas por distintos criterios (legal o científicotécnico)?, 2) ¿Cómo varía la cobertura/uso del suelo (i.e., bosque, uso agrícola y áreas urbanas) en diferentes regiones agroecológicas del pedemonte y la llanura tucumana?, y 3) ¿Cómo varían los porcentajes de la superficie de las unidades de producción agrícola que ocupan las zonas ribereñas comparando los diferentes anchos de amortiguación? Además, discutimos las implicancias que tienen nuestros resultados para un potencial plan de restauración de los bosques de ribera del pedemonte y la llanura de Tucumán.

\section{Materiales y Métodos}

\section{Área de estudio}

El área de estudio se encuentra entre $26^{\circ}-28^{\circ}$ $\mathrm{S}$ y $64^{\circ}-66^{\circ} \mathrm{O}$, incluyendo la mayor parte de la provincia de Tucumán en el noroeste de la Argentina (Figura 1A) correspondiente a zonas de pedemonte y llanura. Decidimos analizar zonas ribereñas ubicadas a una altitud menor a $700 \mathrm{~m}$ sobre el nivel del mar (m s. n. m.) porque esta área es la más modificada de la provincia (Díaz Gómez and Gaspari 2017). Nos concentramos en las llanuras hacia el este, y quedó excluido gran parte del valle de Trancas, en el norte de la provincia, que responde a otras características ambientales y dinámicas de uso de la tierra (Nanni and Grau 2017). Desde el punto de vista hidrológico, el área de estudio incluye dos cuencas principales: a) la mayor parte de la cuenca endorreica del río Salí-Dulce, en la cual el río principal de la cuenca (el río Salí), fluye a través de la provincia de noroeste a sureste y está represado por el Dique El Frontal en la frontera con la provincia de Santiago del Estero. Cabe destacar que la cuenca del río Salí se incluyó entre los sectores prioritarios para la restauración forestal en el plan nacional argentino de restauración de bosques nativos (tinyurl.com/y4sjnpdo), y b) la sub-cuenca del río Urueña, situada en el noreste de Tucumán, la cual forma parte de la cuenca del río Paraná.

Dentrodelárea deestudioestán representadas las ecorregiones de la Selva nublada subtropical de las Yungas (Selva de Yungas) y del Chaco Seco (Brown and Pacheco 2006). La Selva de Yungas es un cinturón estrecho de bosque lluvioso de montaña que oscila entre 400 y 3000 m s. n. m. El clima es cálido y húmedo, con temperaturas medias anuales que oscilan entre 14 y $26^{\circ} \mathrm{C}$ y precipitaciones entre 1000 y 2500 mm/año. Posee un marcado gradiente altitudinal abiótico y biótico (Brown et al. 2001). Este estudio incluye el sector de los bosques de pedemonte que en condiciones naturales presentan árboles caducifolios con dominancia de especies como Tipuana tipu (Tipa blanca) y Enterolobium contortisiliquum (Pacará o Timbó). En particular, el sector de pedemonte en Tucumán presenta una precipitación anual de 1000-1500 mm concentrada durante la estación húmeda, y una estación seca de 6 meses (precipitación de $50 \mathrm{~mm}$ ) que se extiende de junio a noviembre. El Chaco Seco es una vasta llanura fluvial sedimentaria formada por los ríos que corren de noreste a suroeste e incluye gran parte del noroeste de la Argentina y otros países (Gran Chaco sudamericano) (Brown and Pacheco 2006). Las temperaturas medias anuales oscilan entre 19 y $24^{\circ} \mathrm{C}$. La precipitación media anual varía entre 400 y $900 \mathrm{~mm}$; la mayoría de las precipitaciones ocurren en verano (Minneti 1999). La vegetación espontanea está compuesta por bosques secos y praderas segregadas.

El bosque de ribera puede ser muy diferente del adyacente en ambas ecorregiones y se han evidenciado cambios composicionales y estructurales en la vegetación de ribera en general a partir de entre los 20 y $30 \mathrm{~m}$ desde el curso de agua (Pero and Quiroga 2019). En el bosque de ribera de Yungas, las especies dominantes varían según la estratificación altitudinal. Dentro del Chaco Seco, las especies Salix humboldtiana (Sauce criollo) y Tessaria integrifolia (Palo bobo) son comúnmente dominantes en las zonas de ribera. Para el año 1986 no se registró cobertura de pastizales en las zonas de ribera del área de estudio (Díaz Gómez and Gaspari 2017). Cabrera (1976: 8) menciona que en las zonas de ribera del 

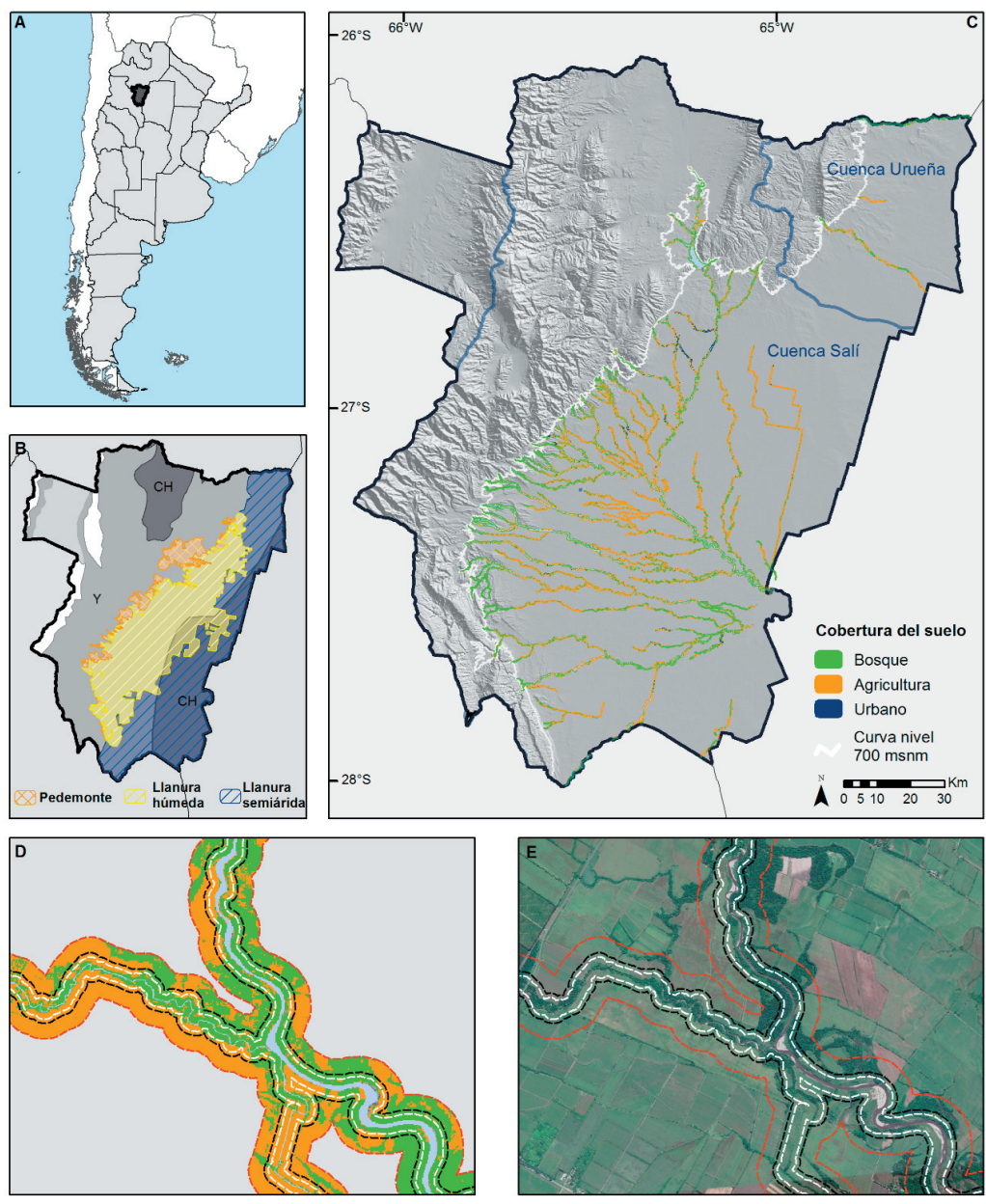

Figura 1. Mapa de clasificación de coberturas de suelo de las zonas ribereñas de la provincia de Tucumán. A: Ubicación en la Argentina. B: Delimitación de las regiones agroecológicas y ecorregiones ( $\mathrm{Y}=$ Yungas, $\mathrm{CH}=$ Chaco Seco). C: Mapa general. Zoom a los diferentes anchos de amortiguación o buffers en un mapa raster (D) y en una imagen en alta resolución (E).

Figure 1. Map of the land cover classification of riparian zones of Tucumán province. A: Location in Argentina. B: Delimitation of the different agroecological regions and ecoregions ( $\mathrm{Y}=$ Yungas, $\quad \mathrm{CH}=$ Western Chaco). C: General map. Zoom to the different buffer strips in the raster map (D) and in a high spatial resolution image (E).

distrito de las Selvas de Transición (el cual cubría gran parte de nuestra área de estudio) se diferencian dos comunidades vegetales: Bosques ribereños de 'tusca', que "ocupan las terrazas bajas inmediatas a los ríos y están formadas principalmente por leguminosas mimosoideas espinosas", con predominio de Acacia aroma (tusca), y Bosquecillos marginales "formados por arbolitos y arbustos que crecen sobre el lecho de inundación de los ríos, con predominio de Tecoma stans (guaran), Tessaria integrifolia, S. humboldtiana, Tessaria dodoneaefolia (chilca) y Baccharis salicifolia (chica)".

Por otro lado, el clima y el paisaje heterogéneo de Tucumán permiten el cultivo de especies agronómicas variadas (Soria et al. 2016). Nuestra área de estudio incluyó las áreas de cultivo de las especies económicamente más importantes para la provincia, incluida la producción de frutas (cítricos, frutilla y arándano) en zonas pedemontanas, caña de azúcar en llanuras húmedas y cultivos de granos (principalmente soja y maíz) y ganadería en las llanuras más secas del este.
Definición del ancho de zonas de amortiguación o buffers ribereños

De acuerdo con la legislación ambiental (Ley Provincial $N^{\circ}$ 8304) en Tucumán, una franja de bosque de ribera está bajo la categoría de protección y se define como una franja de $57 \mathrm{~m}$ de ancho desde el límite del río. Por lo tanto, en nuestro estudio definimos una primera zona de amortiguación de $60 \mathrm{~m}$ de ancho a fin de redondear una propuesta para evaluar la cobertura boscosa de ribera de acuerdo con criterios legales. En segundo lugar, consultamos por correo electrónico (a través de un formulario de Google) a diez especialistas locales (respondieron seis especialistas) de diferentes disciplinas (limnología, ecología de bosques, geología, gestión ambiental) y organismos (academia, gobierno y agencias no gubernamentales) a través de las siguientes preguntas: Si tuviera que recomendar una medida de ancho de bosque de ribera para proteger a partir de donde finaliza el lecho seco de los ríos en la zona pedemontana y de llanura de Tucumán, 
a) ¿Propondría una medida diferente para pedemonte y otra para llanura?, y b) ¿Qué longitud en metros tendría en cada caso o de ser una única medida? El objetivo de la consulta fue establecer un criterio científicotécnico integral para definir el ancho de zonas de amortiguación óptimo para la protección de los bosques de ribera y los SE que proveen. De acuerdo con las respuestas de los/as especialistas consultados/as (Tabla 1 y Material Suplementario), decidimos explorar dos alternativas adicionales de ancho de zonas de amortiguación, una opción de mínima de $100 \mathrm{~m}$ y una segunda de máxima de $250 \mathrm{~m}$, indistintamente para pedemonte y llanura. La opción de $100 \mathrm{~m}$ se seleccionó debido a que la mayor parte de los/as especialistas propusieron esa longitud como un medida mínima para la protección de las riberas. La opción de 250 m fue propuesta por una especialista siguiendo un criterio geomorfológico para proteger el cauce fluvial y mantener su dinámica espaciotemporal, priorizando el servicio ecosistémico de estabilización de riberas. En consecuencia, evaluamos 3 zonas de amortiguación ribereñas de diferentes anchos (60, 100 y 250 m). Para delimitar de forma precisa las zonas de amortiguación fue necesario definir una línea de costa o ribera desde la cual iniciarla. Para esto se crearon polígonos de los cauces de los ríos y se generó una capa actualizada de la red fluvial provincial. La delimitación de estos polígonos se llevó a cabo de forma manual a través de fotointerpretación en un mosaico del área de estudio compuesto por 48 imágenes satelitales Sentinel, el cual abarca el período julio-septiembre de 2018, por ser las fechas del año con la mayor disponibilidad de imágenes sin nubes. Los cauces delimitados incluyeron tanto el canal activo (húmedo) como las llanuras de inundación sin vegetación y márgenes secos, utilizando como base la capa oficial de la red fluvial provincial provista por la Dirección Provincial de Recursos Hídricos.

\section{Clasificación de coberturas del suelo}

La selección de imágenes y los mapas de cobertura/usos del suelo terrestre se realizaron a través de Google Earth Engine (GEE) (earthengine.google.com/), una plataforma gratuita que facilita acceder $y$ procesar remotamente grandes conjuntos de datos geoespaciales (Gorelick et al. 2017). Trabajamos con imágenes de la misión Sentinel del Programa Copernicus (Agencia Espacial Europea, www.copernicus.eu/en), con un tiempo de revisita de cinco días y 13 bandas espectrales de resolución espacial de 10,20 y $60 \mathrm{~m}$. Utilizamos productos derivados del Sentinel-2 MultiSpectral Instrument (MSI) Nivel 1-C, compuestos de mosaicos de $100 \mathrm{~km}^{2}$ (orto-imágenes en proyección UTM / WGS84), $\mathrm{y}$ en los que se proporcionan mediciones radiométricas por píxel al tope de la atmósfera (TOA) (ESA 2015).

Para obtener mapas de cobertura/uso del suelo, construimos un mosaico con los valores de la mediana estadística en cada píxel para cada banda correspondientes al período julioseptiembre de 2018. Preferimos este período del año por el menor porcentaje de nubes respecto de otros meses del año. La presencia

Tabla 1. Respuestas resumidas de los/las especialistas consultados/as sobre el ancho óptimo para zonas de amortiguación ribereña.

Table 1. Summarized answers of the consulted specialists on the optimal width for riparian buffer zones.

\begin{tabular}{|c|c|c|}
\hline Especialista & Disciplina/ámbito laboral & Respuesta \\
\hline 1 & Limnología/Academia & $\mathrm{Si}, 57 \mathrm{~m}$ en llanura, $50 \mathrm{~m}$ en pedemonte \\
\hline 2 & Geología/Academia & $\begin{array}{l}\text { "El ancho debería ser de entre } 250 \text { y } 300 \mathrm{~m} \text { " .. "podría establecerse } \\
\text { un ancho mayor en el piedemonte }(300 \mathrm{~m}) \text { y entre } 200 \text { y } 250 \mathrm{~m} \text { en } \\
\text { la llanura" }\end{array}$ \\
\hline 3 & $\begin{array}{l}\text { Gestión Ambiental/Organismo } \\
\text { No Gubernamental }\end{array}$ & $\begin{array}{l}\text { "Si se debiera asignar un ancho a los bosques de ribera, no debería } \\
\text { ser menos a } 100 \mathrm{~m} \text { " }\end{array}$ \\
\hline 4 & Limnología/Academia & "En el primer caso propondría $>30 \mathrm{~m}$ y en el segundo $>100 \mathrm{~m}$ " \\
\hline 5 & Ecología de bosques/Academia & $\begin{array}{l}\text { "Tomaría una medida no menor a } 150 \text { m en áreas de llanura } \\
\text { considerando el lecho de río seco" }\end{array}$ \\
\hline 6 & Ecología de bosques/Academia & $\begin{array}{l}\text { "propongo una medida de buffer no menor a } 70 \mathrm{~m} \text { en pedemonte } \\
\text { y de } 100 \mathrm{~m} \text { en llanura" }\end{array}$ \\
\hline
\end{tabular}


de nubes no permite observar con claridad las escenas ni clasificarlas correctamente. Trabajamos con imágenes con una cobertura total de nubosidad igual o menor al 6\%, ya que no se encontraron suficientes imágenes con un umbral menor de nubosidad para cubrir la totalidad del área de estudio. Aplicamos un filtro de nubosidad, sobre cada escena, el cual corresponde a un algoritmo proporcionado por GEE, disponible en tinyurl.com/y49qvmue. Elegimos la mediana porque resume la información de todas las imágenes y es poco afectada por los valores extremos que podrían existir en los datos. Extrajimos las zonas ribereñas y adyacentes a estas de la imagen mediana usando una distancia de 2 kilómetros $(\mathrm{km})$ de ancho desde una línea que cruza el centro de los arroyos y ríos; esta extracción de un área más amplia obedece a razones prácticas para luego clasificar los usos de suelo dentro de esa zona. Aplicar la distancia de $2 \mathrm{~km}$ para clasificar la cobertura de la tierra nos permitió disponer de un área suficientemente grande en donde tomar muestras para clasificar; esto resulta en clasificaciones más precisas. Todos los cálculos de superficie refieren a las de zonas de amortiguación.

La clasificación se realizó utilizando el clasificador Random Forest (RF) (Breiman 2001), una técnica de entrenamiento supervisado y no paramétrico. RF trabaja creando varios árboles de decisión y calcula la respuesta en función del resultado de todos los árboles, lo que resulta en clasificaciones altamente precisas (Rodríguez-Galiano et al. 2012). Para clasificar, ajustamos 20 árboles y consideramos un subconjunto aleatorio de todas las variables para dividir cada nodo de árbol, que en GEE es la raíz cuadrada del número de variables en los datos. Para discriminar las clases bosque, agricultura, urbano, agua y sombra (corresponde a sombras producidas por el recorte de nubes en la imagen), clasificamos la imagen usando las bandas 2, 3, 4, 5, 6, 7, 8, 8A, 11 y 12, y una banda de Índice de Vegetación de Diferencia Normalizada (IVN o NDVI por sus siglas en inglés) calculada utilizando las bandas del Rojo e Infra-rojo cercano (NIR). Se tomaron muestras en forma de polígonos para entrenamiento y puntos para validación de cada clase. El programa (GEE) toma el valor de cada píxel dentro de los polígonos para el entrenamiento. Se tomó como referencia el Mapa Nacional de Cultivos 2018/2019 (de Abelleyra et al. 2019) y puntos relevados en el campo durante el año 2018 para identificar las clases de cobertura en aquellos casos de difícil diferenciación en la imagen. La clasificación fue posteriormente realizada sobre la imagen correspondiente a la mediana del mosaico Sentinel. Para la validación se tomaron sólo puntos debido a que al utilizar polígonos existe auto-correlación espacial entre los puntos internos de cada polígono y por lo tanto dejan de ser muestras independientes (Olofsson et al. 2013). Se tomaron 100 puntos de validación por clase, teniendo en cuenta que el mínimo de muestras de validación por clase de cobertura deberían ser 50 puntos (Congalton and Green 2008). La única excepción fue la clase sombra, la cual ocupa escasa superficie en el mosaico, pero consideramos importante separarla de las demás coberturas ya que de lo contrario se confundía con algunos parches de cultivos quemados, como suele ocurrir en parches donde ya se cosechó la caña de azúcar. La cantidad de polígonos de entrenamiento y puntos de validación por cada clase fueron los siguientes: bosque, 33 polígonos y 100 puntos, cultivos 48 polígonos y 100 puntos, urbano 30 polígonos y 100 puntos, agua 30 polígonos y 100 puntos, sombra 6 polígonos y 12 puntos. Se utilizaron la exactitud total del mapa, del usuario y del productor por cada clase como métricas para la validación reportándolas en una matriz de confusión (Pontius and Millones 2011; Foody 2020).

\section{Delimitación de regiones agroecológicas}

Delimitamos tres regiones agroecológicas principales (Figura 1B) siguiendo un mapa local de distribución de cultivos de los años 2014-2015 (Soria et al. 2016): a) la región de pedemonte, ubicada en las zonas pedemontanas de las montañas principales, coincidiendo con el bosque pedemontano de Yungas donde se ubica principalmente el cultivo de cítricos y arándanos, b) la región de la llanura húmeda, que se encuentra en el centro de la llanura correspondiente a zonas más secas de las Yungas y la transición al bosque chaqueño del Chaco Seco y cuyo principal cultivo es el de caña de azúcar; y la región de la llanura seca, ubicada en los márgenes de la llanura provincial hacia el este y donde predomina el cultivo de granos.

\section{Cálculo de superficies de cobertura del suelo en zonas ribereñas y unidades catastrales}

Para evaluar la frecuencia de las unidades de producción agrícola (UPA) (definidas por 
registros de lotes catastrales) con diferentes proporciones incluidas en la zona ribereña, trabajamos sobre una capa catastral oficial de la provincia de Tucumán. Realizamos una intersección entre la capa catastral y las zonas de amortiguación ribereña para identificar las UPA adyacentes a los ríos. Fueron excluidos de los análisis aquellos lotes catastrales de menos de 1 hectárea de superficie. Las diferentes capas (coberturas de suelo, ríos, zonas de amortiguación, sectores agrícolas y lotes catastrales) se solaparon y cortaron para calcular las diferentes superficies. Todos los análisis espaciales se realizaron con herramientas estándar del software libre y gratuito de sistemas de información geográfica QGis (QGis 2014, versión 2.6.0).

\section{Resultados}

Situación general: coberturas del suelo y zonas
ribereñas

El área total incluida en las diferentes zonas de amortiguación ribereñas fue: 32111 ha para $60 \mathrm{~m}$ de ancho, 52223 ha para $100 \mathrm{~m}$ de ancho y 121654 ha para $250 \mathrm{~m}$ de ancho. La evaluación de la clasificación mostró que el clasificador RF discriminó con precisión las diferentes coberturas del suelo, alcanzando una exactitud total de 0.87 (87\% de muestras clasificadas correctamente) (ver matriz de confusión, Tabla 2). La ubicación de los diferentes usos del suelo y superficies boscosas en las diferentes zonas de amortiguación se

Tabla 2. Matriz de confusión.

Table 2. Confusion matrix.

\begin{tabular}{|c|c|c|c|c|c|c|c|c|}
\hline $\begin{array}{l}\text { Datos de referencia/Datos } \\
\text { clasificados }\end{array}$ & Bosque & Cultivos & Urbano & Agua & Sombra & $\begin{array}{l}\text { Total } \\
\text { filas }\end{array}$ & $\begin{array}{l}\text { Exactitud } \\
\text { de usuario }\end{array}$ & $\begin{array}{c}\text { Exactitud de } \\
\text { usuario por } \\
\text { clase }(\%)\end{array}$ \\
\hline Bosque & 94 & 6 & 0 & 0 & 0 & 100 & 0.94 & 94 \\
\hline Cultivos & 16 & 79 & 3 & 2 & 0 & 100 & 0.79 & 79 \\
\hline Urbano & 0 & 13 & 87 & 0 & 0 & 100 & 0.87 & 87 \\
\hline Agua & 5 & 1 & 5 & 89 & 0 & 100 & 0.89 & 89 \\
\hline Sombra & 3 & 0 & 0 & 0 & 9 & 12 & 0.75 & 75 \\
\hline Total columnas & 118 & 99 & 95 & 91 & 9 & 412 & & \\
\hline Exactitud de productor & 0.80 & 0.80 & 0.92 & 0.98 & 1.00 & & & \\
\hline Exactitud de productor (\%) & 80 & 80 & 92 & 98 & 100 & & & \\
\hline Exactitud total & & & & & & & 0.87 & 87 \\
\hline
\end{tabular}

Tabla 3. Superficies de las coberturas de suelo (ha) de las diferentes clases (bosque, agricultura, urbano, otros) dentro de las diferentes alternativas de zona de amortiguación $(60,100$, y $250 \mathrm{~m}$ ) en los diferentes sectores agrícolas (pedemonte, llanura húmeda, llanura seca).

Table 3. Land covers area (ha) of the different classes (forest, agriculture, urban, others) within the different buffers strip $(60,100$ and $250 \mathrm{~m}$ ) in the different agricultural zones (foothill, wet plain, dry plain).

\begin{tabular}{|c|c|c|c|c|c|c|c|c|c|c|c|c|}
\hline \multirow{2}{*}{$\begin{array}{l}\text { Zona de } \\
\text { amortiguación }\end{array}$} & \multicolumn{3}{|c|}{ Pedemonte } & \multicolumn{3}{|c|}{ Llanura húmeda } & \multicolumn{3}{|c|}{ Llanura seca } & \multicolumn{3}{|c|}{ Totales } \\
\hline & $60 \mathrm{~m}$ & $100 \mathrm{~m}$ & $250 \mathrm{~m}$ & $60 \mathrm{~m}$ & $100 \mathrm{~m}$ & $250 \mathrm{~m}$ & $60 \mathrm{~m}$ & $100 \mathrm{~m}$ & $250 \mathrm{~m}$ & $60 \mathrm{~m}$ & $100 \mathrm{~m}$ & $250 \mathrm{~m}$ \\
\hline \multirow[t]{2}{*}{ Bosque } & 6290 ha & 10009 & 22232 & 7101 & 10179 & 17906 & 5055 & 8001 & 17455 & 18446 & 28189 & 57593 \\
\hline & $(75.3 \%)$ & $(74 \%)$ & $(70.8 \%)$ & $(46.4 \%)$ & $(40.9 \%)$ & $(31.0 \%)$ & $(59.7 \%)$ & $(57.9 \%)$ & $(53.8 \%)$ & & & \\
\hline \multirow[t]{2}{*}{ Agricultura } & 1628 ha & 2814 & 7491 & 7503 & 13799 & 38049 & 2967 & 5268 & 13942 & 12098 & 21881 & 59482 \\
\hline & $(19.5 \%)$ & $(20.8 \%)$ & $(23.9 \%)$ & $(49.1 \%)$ & $(55.5 \%)$ & $(65.8 \%)$ & $(35.1 \%)$ & $(38.1 \%)$ & $(42.9 \%)$ & & & \\
\hline \multirow[t]{2}{*}{ Urbano } & 331 ha & 544 & 1397 & 281 & 443 & 1166 & 146 & 196 & 353 & 758 & 1183 & 2916 \\
\hline & $(4.0 \%)$ & $(4.0 \%)$ & $(4.5 \%)$ & $(1.8 \%)$ & $(1.8 \%)$ & $(2.0 \%)$ & $(1.7 \%)$ & $(1.4 \%)$ & $(1.1 \%)$ & & & \\
\hline \multirow{2}{*}{$\begin{array}{l}\text { Otros (agua, } \\
\text { sombra) }\end{array}$} & 108 ha & 145 & 259 & 406 & 461 & 692 & 295 & 364 & 712 & 809 & 970 & 1663 \\
\hline & $(1.3 \%)$ & $(1.2 \%)$ & $(0.8 \%)$ & $(2.7 \%)$ & $(1.9 \%)$ & $(1.2 \%)$ & $(3.5 \%)$ & $(2.6 \%)$ & $(2.2 \%)$ & & & \\
\hline Total (ha) & 8357 & 13512 & 31379 & 15291 & 24882 & 57813 & 8463 & 13829 & 32462 & & & \\
\hline
\end{tabular}


puede observar en el mapa producto de la clasificación (Figura 1C, D y E). En el total del área de estudio encontramos que dentro de la zona de amortiguación de $60 \mathrm{~m}$ de ancho, $40 \%$ del área está ocupada por usos del suelo, principalmente agricultura (38\%) y en menor proporción por urbanización ( $2 \%)$, totalizando $\sim 12800$ ha de superficie sin bosque de ribera (Tabla 3). Dentro de las zonas de amortiguación definidas por criterios científico-técnicos aumentó el porcentaje de las zonas ribereñas transformadas en agricultura. En el ancho de $100 \mathrm{~m}$, los porcentajes de cobertura del suelo son en su mayoría similares al ancho legal, pero en valores absolutos, el área ocupada por la agricultura es casi el doble en comparación con aquel ancho. En el caso de la zona de amortiguación de $250 \mathrm{~m}$ de ancho, el porcentaje de zonas ribereñas cubiertas por la agricultura es mayor (51\%) que en las zonas de amortiguación más estrechas, excediendo al área boscosa. La cobertura del área ocupada por uso urbano mantiene su porcentaje casi sin variaciones entre las distintas alternativas de zonas de amortiguación.

\section{Segmentación por zonas agroecológicas}

Se encontraron diferentes patrones de distribución de la cobertura de suelo y superficie en las zonas ribereñas entre las diferentes regiones agroecológicas (Tabla 3 ). Dentro de la región del pedemonte encontramos la mayor superficie de bosque de ribera, con un $23.4 \%$ (1959 ha) de su superficie dentro de esta región, ocupada por otros usos del suelo (agrícola y urbano) dentro de los 60 $\mathrm{m}$ de zona de amortiguación. La región de la llanura húmeda presenta la menor cobertura de bosque de ribera con un 50.9\% (7784 ha) de la zona ribereña de esta región, ocupada por agricultura y uso urbano considerando los 60 $\mathrm{m}$ de amortiguación. La región de la llanura seca presenta una situación intermedia de superficie boscosa con el $36.8 \%$ de las zonas ribereñas de esta región, cubiertas por usos del suelo agrícola y urbano (3113 ha) también considerando los $60 \mathrm{~m}$ de amortiguación. El patrón de superficies de bosque y usos del suelo no cambia con las diferentes distancias de amortiguación, observando en general la menor superficie boscosa en la región de la llanura húmeda, una situación intermedia en la región de la llanura seca y la mayor superficie de bosque en la región del pedemonte. La categoría urbana cubre mucha menos superficie en las diferentes regiones agroecológicas que las otras clases de cobertura del suelo. Sin embargo, la región del pedemonte mostró una cobertura del suelo urbana ligeramente mayor en comparación con las demás regiones agroecológicas (Tabla 3).

\section{Zona ribereña y unidades de producción agrícola}

Según el catastro de la provincia de Tucumán, un total de 10315 unidades de producción agrícola (UPA) se encontraron adyacentes a
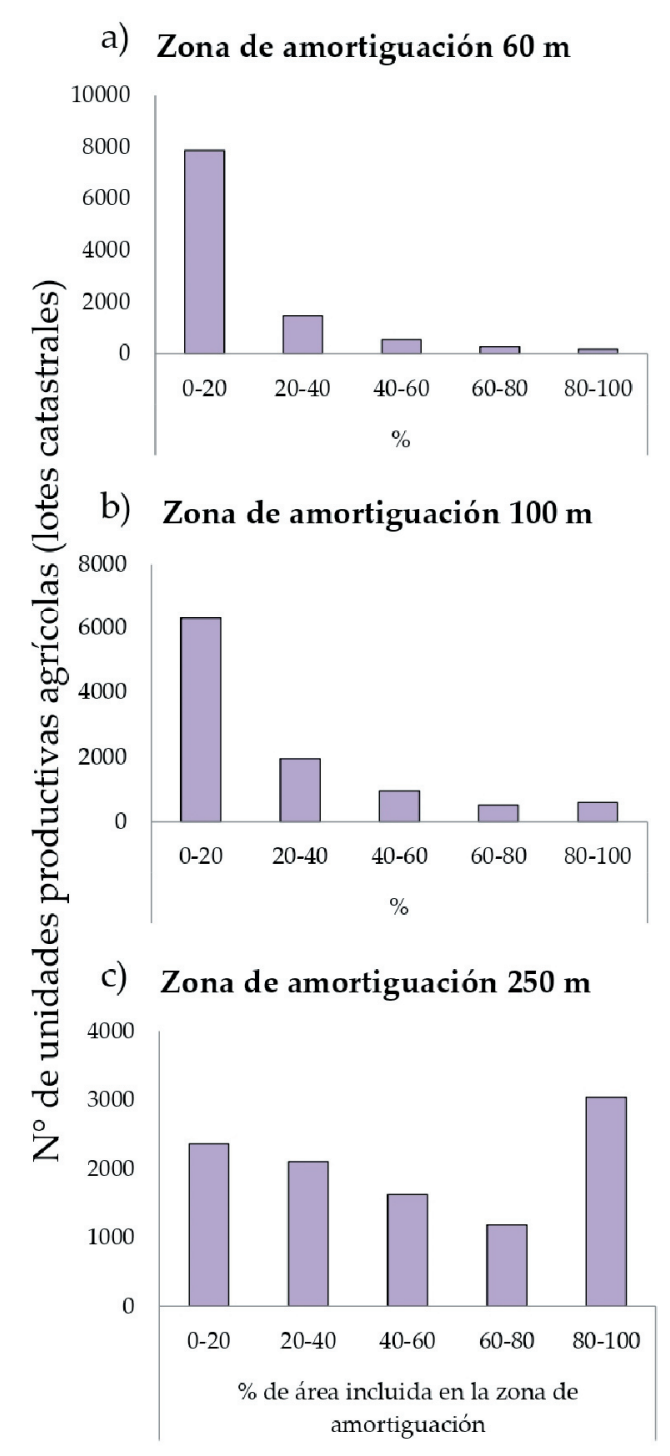

Figura 2. Número de unidades productivas agrícolas (UPA) (lotes catastrales) con diferentes porcentajes de superficie $(0-20 ; 20-40 ; 40-60 ; 60-80 ; 80-100 \%)$ incluida en la zona ribereña según las distancias alternativas de amortiguación: a) $60 \mathrm{~m}$; b) $100 \mathrm{~m}$; c) $250 \mathrm{~m}$.

Figure 2. Number of agronomic productive units (cadastral units) with different percentage of area (0-20; $20-40 ; 40-60 ; 60-80 ; 80-100 \%)$ included in the riparian zone using alternative buffer distances: a) $60 \mathrm{~m}$; b) 100 m; c) $250 \mathrm{~m}$. 
los ríos e incluían alguna proporción de la zona ribereña. Al analizar los casos de zonas de amortiguación de 60 y 100 m, observamos que la gran mayoría de las UPA adyacentes a los ríos están ocupadas en menos del 20\% de su área por la zona ribereña. Este patrón se modifica enormemente al analizar la zona de amortiguación de $250 \mathrm{~m}$, con la mayoría de las UPA incluidas en más de un $80 \%$ de su área dentro de la zona ribereña (Figura 2).

\section{Discusión}

En general, nuestros resultados revelaron que el pedemonte y las llanuras de Tucumán presentan entre 12856 y 62398 ha ocupadas por usos del suelo diferentes al bosque de ribera, según el ancho de zona ribereña de amortiguación de 60 y 250 m respectivamente. Los usos agrícolas cubren el mayor porcentaje de aquellas zonas que potencialmente podrán ser objetivo de las iniciativas de restauración. El porcentaje de cobertura de los distintos usos de suelo que cubren las zonas ribereñas varió entre las diferentes regiones agroecológicas. Es probable que las diferencias topográficas y climáticas de cada región hayan influido en estas diferencias. Por ejemplo, la mayor pendiente del pedemonte y la mayor sequedad en la llanura seca limitaron el avance agrícola en estas regiones, al contrario de lo observado en la llanura húmeda. Por lo tanto, consideramos que se deberían realizar y asignar diferentes esfuerzos y recursos para que la RE de estas zonas sea más eficiente y efectiva.

Los diferentes criterios de ancho de zonas de amortiguación ribereñas mostraron diversas necesidades de restauración. El ancho definido por criterios legales tuvo $40 \%$ de su superficie total ocupada por usos del suelo antrópicos ( 12800 ha), superficie cuya restauración sería necesaria para poder cumplir con el requisito legal de protección ribereña provincial. Para alcanzar un total de cobertura de bosque de ribera dentro del ancho de amortiguación mínimo sugerido por los criterios científico-técnicos, sería necesario restaurar aproximadamente 10000 ha más que para el requisito legal actual. En el plan de restauración nacional de la Argentina se propone una tasa de restauración de 20000 ha por año para 2023. Entre algunos de los objetivos de restauración global, por ejemplo, el de la Asociación Mundial para la Restauración del Paisaje Forestal se propone restaurar 400 millones de ha de bosque ( $\underline{w}$ .forestlandscaperestoration.org), y el desafío de Bonn (www.bonnchallenge.org, que recibe contribuciones y apoyo de la plataforma regional Iniciativa 20×20 en Latinoamérica y el Caribe [initiative20x20.org]), tiene como metas la restauración de bosques en 150 millones de ha para el año 2020 y 350 millones de ha para el año 2030. Consideramos que la superficie de bosque a restaurar necesaria para cumplir con el requisito legal de la provincia de Tucumán (12800 ha) es acorde a las expectativas de las mencionadas metas de restauración nacionales y globales. Un potencial plan de restauración que contemple la RE de aquellas superficies podría cumplirse en un mediano plazo (5 años) a una tasa de 2000 ha restauradas por año. Sin embargo, se deberán contemplar y analizar en detalle otros aspectos locales como la propiedad de la tierra, la superficie cultivada total de cada lote y las expectativas o resistencias de productores agrícolas con respecto a proyectos de RE en las riberas de sus propiedades.

La factibilidad de los objetivos de restauración propuestos está respaldada en líneas generales por el hecho de que la gran mayoría de las UPA incluyen menos de $20 \%$ de su superficie en la zona de amortiguación ribereña más estrecha $(60 \mathrm{~m})$. No obstante, 2000 lotes catastrales presentaron más de $20 \%$ de su superficie en la zona ribereña. Para aquellos casos en los cuales sí se ven afectadas las UPA en mayor porcentaje, proponemos evaluar alternativas que incluyan, por ejemplo, menores porcentajes de RE y hacer foco en zonas críticas (i.e., llanuras de inundación o márgenes erosivos). En contraste, el uso de la distancia de amortiguación de 250 m cambia el panorama en términos prácticos y de factibilidad. La aplicación de esta distancia, implica que la mayoría de las UPA adyacentes a los ríos deberían disminuir en más de la mitad su superficie con uso agrícola. En nuestra opinión, esta situación podría representar una limitación importante para un plan de restauración factible. Sin embargo, creemos que un plan de restauración de riberas en distancias de 60 o 100 m podría complementarse, logrando que los bosques existentes en la llanura tucumana que actualmente se encuentran dentro de la zona de amortiguación de 250 m, se incluyan en categorías de protección de acuerdo con la zonificación de la Ley Nacional N²6331 de bosques nativos. Por ejemplo, promoviendo zona amarilla para áreas ubicadas a mayor distancia de los ríos, permitiendo un manejo forestal y/o silvopastoril, y zona roja para 
conservación de aquellas áreas más cercanas a los ríos con distancias inferiores a los 100 m. De esta manera se podría evitar la futura deforestación de estas zonas ribereñas hasta $250 \mathrm{~m}$ (actualmente en categoría verde) a la vez que se recuperan zonas de ribera que hoy no tienen bosque dentro de los 60 o 100 $\mathrm{m}$ de ancho. Aquellos propietarios cuyos lotes contienen alguna superficie de bosque pueden acceder al apoyo y financiamiento de la Ley 26331 para conservar estos bosques, mejorar su calidad e incluso restaurar las zonas aledañas a los mismos. Sin embargo, no existe en la actualidad una legislación provincial o nacional que contemple presupuestos mínimos para la restauración de sectores deforestados en los cuales no existe vegetación boscosa en el presente (Straccia and Isla 2020). Teniendo en cuenta las mencionadas necesidades locales y la tendencia mundial y regional a la priorización de políticas públicas de conservación y recuperación de ambientes naturales entre ellos, humedales y bosques (e.g., ODS 2030), sería importante avanzar hacía una legislación específica para RE en la Argentina.

Los proyectos de RE a plantear deberán contemplar opciones de restauración adecuadas a aquellas situaciones en las cuales la misma conlleve un compromiso importante para la producción agrícola local. Es necesario pensar estrategias de compensación y herramientas legales que faciliten y promocionen la opción de restaurar los márgenes de ribera de las propiedades privadas lindantes a los cursos de agua. Además, es importante considerar que las zonas ribereñas son particularmente irregulares para la producción agronómica porque comúnmente se ven afectadas por procesos de inundación y erosión (Naiman et al. 2005). Por ende, se podría esperar que la $\mathrm{RE}$ de las zonas ribereñas resulte en una menor perdida de producción agrícola (en términos de productividad) considerando el mediano plazo comparado con zonas interiores más alejadas de la ribera sometidas a menores disturbios. En la actualidad, la problemática de las inundaciones y de la erosión de los márgenes de ribera que atraviesan terrenos productivos es motivo de preocupación para los productores agrícolas locales (Comisión de Emergencia: Informe Técnico 2017, y comunicaciones personales de productores locales). Esta preocupación llevó a diferentes grupos de productores y al gobierno provincial a iniciar proyectos de recuperación de bosques y vegetación de ribera para el mejoramiento de los sistemas de drenaje principalmente en el área cañera (llanura húmeda) (Fortini and Apud, comunicación personal). La preocupación por las consecuencias de procesos erosivos y de inundación de los márgenes de ribera y la capacidad de los bosques de ribera de proveer SE de estabilización de márgenes y regulación de inundaciones (Riis et al. 2020) pueden ser motivaciones importantes para comprometer a los productores en la concreción de los proyectos de RE de riberas y promocionar los beneficios de los mismos. Sería fundamental llevar acabo un abordaje socioecológico (Roulier et al. 2020) para elaborar los proyectos de RE de manera de incluir en los mismos las visiones, percepciones y expectativas de los productores locales y demás actores sociales involucrados (e.g., funcionarios públicos).

Contemplando el contexto regional y local consideramos que el mapeo realizado en nuestro estudio será una herramienta útil para focalizar proyectos de RE de un ecosistema vulnerable y que provee SE esenciales para abordar la problemática de inundaciones y erosión de cauces. La metodología utilizada a través de la plataforma Google Earth Engine permitió el mapeo detallado, rápido y a gran escala de áreas ribereñas. Este tipo de técnicas podría usarse para mapear otros sectores de la Argentina o de América del Sur. Sin embargo, en el caso de áreas boscosas densas y diversas que poseen bosques con diferente estructura y composición, las técnicas de teledetección no permiten distinguir con facilidad estos tipos de vegetación a escalas espaciales regionales (Macfarlane et al. 2016). Por lo tanto, a escalas locales (sitios o tramos de río) se deben realizar muestreos más detallados para evaluar la calidad del bosque (e.g., presencia de especies invasoras, estructura de estratos, presencia de ganado) (Sirombra and Mesa 2012) e identificar áreas que también necesitan algún nivel de restauración (Almeida et al. 2019). A escalas locales de tramo de río o de lotes agrícolas será importante evaluar las características de los sectores a restaurar (e.g., características geomorfológicas y tipos de suelo) para evaluar las estrategias de RE a implementar. Por ejemplo, considerando la heterogeneidad física y biológica de los ecosistemas de ribera en sitios de referencia (Pero and Quiroga 2019), en los sectores de llanura de inundación podría ser adecuada una restauración pasiva pero monitoreada, en la cual las especies típicas de estos microambientes se establecen con mayor facilidad (e.g, Salix humbodtiana o Tessaria 
integrifolia), mientras que en las terrazas fluviales más elevadas sería necesaria una intervención activa con plantación de especies particulares para cada ecorregión (e.g., Tipuana tipa o Enterolobium contortisiliquum en pedemonte de Yungas y Prosopis alba y Celtis ehrenbergiana en Chaco semiárido). Teniendo en cuenta que el objetivo de la RE es restaurar ecosistemas a un estado 'natural' previo a las transformaciones humanas, se podría discutir cuál fue ese estado en las riberas del Chaco semiárido. Es sabido que tanto en la actualidad como en el pasado la ecorregión del Chaco Seco incluye pastizales de tipo sabanas con alternancia de sectores boscosos (Fernández et al. 2020). Sin embargo, los antecedentes para el área de estudio no han registrado coberturas de pastizales naturales en las riberas para el año 1986 (Díaz Gómez and Gaspari 2017) y 1976 (Cabrera 1976). Por otro lado, en zonas del Chaco semiárido se ha registrado una recuperación de ciertas características funcionales del bosque en parches de bosques secundarios luego de entre 10 y 15 años de abandonada la actividad agrícola (Basualdo et al. 2019). Por lo tanto, sería interesante evaluar distintas opciones de restauración, entre las cuales una intervención mixta (pasiva en llanuras de inundación y activa en terrazas fluviales con posterior monitoreo) podría ser adecuada y eficiente para el área de estudio.

Las zonas ribereñas en el pedemonte y llanura de Tucumán tuvieron diferentes niveles de modificación de la cobertura dentro de la variedad de paisajes agroecológicos analizados. Mapear y evaluar el estado y la composición de las zonas ribereñas a través de diferentes unidades de paisaje permitirán llevar a cabo programas de RE más precisos y eficientes (Strassburg et al. 2019). Además, la información obtenida en este trabajo será útil como base para estimar los beneficios y compromisos de la restauración del bosque de ribera en términos de SE y cambios en el uso del suelo (Barral et al. 2015). Consideramos que el mapeo realizado y los datos generados en este estudio serán una herramienta valiosa para planificar e implementar potenciales proyectos de restauración y que representan un aporte valioso de información relevante a nivel regional que se puede ir actualizando regularmente.

Agradecimientos. Agradecemos a P. Powell y J. Foguet por ayudarnos durante el procedimiento de clasificación de coberturas de suelo; a E. Guido, E. Domínguez, H. Fernández, S. Malizia y K. García por responder a nuestra consulta sobre el ancho óptimo de buffers de ribera; y a la Dirección de Recursos Hídricos del gobierno de Tucumán por facilitarnos la capa oficial de la red fluvial provincial. Los comentarios y sugerencias de dos revisores anónimos/as ciertamente han mejorado el manuscrito final. Este estudio fue apoyado por becas del CONICET (Consejo Nacional de Investigaciones Científicas y Técnicas, Argentina) y el siguiente subsidio: P-UE CONICET 0099.

\section{REFERENCIAS}

de Abelleyra, D., S. Banchero, S. Verón, J. Mosciaro, and J. Volante. 2019. Mapa Nacional de Cultivos Campaña $2018 / 2019$. Colección 1, versión 1. Ministerio de Agricultura, Ganadería y Pesca, Instituto Nacional de Tecnología Agropecuaria (INTA), Presidencia de la Nación, Argentina. URL: tinyurl.com/yypxnzne.

Almeida, D. R. A., C. S. Stark, R. Chazdon, B. W. Nelson, R. G. Cesar, P. Meli, E. B. Gorgens, M. M. Duarte, R. Valbuena, V. S. Moreno, A. F. Mendes, N. Amazonas, N. B. Gonçalves, C. A. Silva, J. Schietti, and P. H. S. Brancalion. 2019. The effectiveness of lidar remote sensing for monitoring forest cover attributes and landscape restoration. Forest Ecology and Management 438:34-43. https://doi.org/10.1016/j.foreco.2019.02.002.

Aronson, J., J. N. Blignaut, and T. B. Aronson. 2017. Conceptual frameworks and references for landscape-scale restoration: reflecting back and looking forward. Ann Missouri Bot Gar 102:188-200. https://doi.org/10.3417/2017003.

Basualdo, M., N. Huykman, J. N. Volante, J. M. Paruelo, and G. Piñeiro. 2019. Lost forest? Ecosystems functional changes occurring after agricultural abandonment and forest recovery in the semiarid Chaco forest. Science of the Total Environment 650:1537-1546. https://doi.org/10.1016/j.scitotenv.2018.09.001.

Barral, M. P., J. M. Rey Benayas, P. Meli, and N. O. Maceira. 2015. Quantifying the impacts of ecological restoration on biodiversity and ecosystem services in agroecosystems: A global meta-analysis. Agriculture, Ecosystems and Environment 202:223-231. https://doi.org/10.1016/j.agee.2015.01.009.

Breiman, L. 2001. Random forest. Machine Learning 45:5-32. https://doi.org/10.1023/A:1010933404324. https://doi.org/ 10.1023/A:1017934522171.

Brown, A. D., H. R. Grau, L. R. Malizia, and A. Grau. 2001. Argentina. Pp. 623-659 en M. Kapelle and A. D. Brown (eds.). Bosques Nublados del Neotrópico. INBio. Heredia, Costa Rica.

Brown, A. D., and S. Pacheco. 2006. Propuesta de actualización del mapa ecorregional de la Argentina. Pp. 28-31 en A. Brown, U. Martínez Ortiz, M. Acerbi, and J. Corcuera (eds.). La situación ambiental argentina 2005. Fundación Vida Silvestre, Buenos Aires, Argentina.

Cabrera, A. L. 1976. Regiones fitogeográficas argentinas. Editorial ACME, Buenos Aires, Argentina. 
Capon, S. J., L. E. Chambers, R. Mac Nally, R. J. Naiman, P. Davies, N. Marshall, J. Pittock, M. Reid, T. Capon, M. Douglas, J. Catford, D. S. Baldwin, M. Stewardson, J. Roberts, M. Parsons, and S. E. Williams. 2013. Riparian ecosystems in the 21th century: hotspot for climate change adaptation? Ecosystems 16:359-381. https://doi.org/10.1007/s10021013-9656-1.

Congalton, R. G., and K. Green. 2008. Assessing the accuracy of remotely sensed data. Principles and practices. 2nd Edition, CRC Press, Florida, USA. https://doi.org/10.1201/9781420055139.

Díaz, A. M. 2018. Las inundaciones en la provincia de Tucumán: una problemática que se repite. Jornadas Platenses de Geografía y XX Jornadas de Investigación y Enseñanza de la Geografía, La Plata, 17-19 Octubre 2018. Universidad Nacional de La Plata, La Plata, Argentina.

Díaz Gómez, A. R., and F. J. Gaspari. 2017. Cambio de cobertura y uso de suelo en la zona ribereña en cuencas subtropicales del noroeste argentino. Quebracho 25(1,2):28-39.

ESA. 2015. Sentinel-2 User Handbook. Revision 2. ESA Standard Document. ESA, Paris.

Fernández, D. P., M. Baumann, G. Baldi, R. N. Banegas, S. Bravo, N. I. Gasparri, M. Lucherini, S. Marinaro, S. A. Nanni, J. A. Nasca, T. Tessi, and H. R. Grau. 2020. Grasslands and Open Savannas of the Dry Chaco. Pp. 562-576 en M. Goldstein and D. DellaSala (eds.). Encyclopedia of the World's Biomes, 1a edición. Elsevier. https://doi.org/ 10.1016/B978-0-12-409548-9.12094-9.

Foody, G. M. 2020. Explaining the unsuitability of the kappa coefficient in the assessment and comparison of the accuracy of thematic maps obtained by image classification. Remote Sensing of Environment 239:111630. https: //doi.org/10.1016/j.rse.2019.111630.

Gasparri, N. I. 2016. The transformation of Land-Use Competition in the Argentinean Dry Chaco Between 1975 and 2015. Pp. 59-73 en J. Niewöhner, A. Bruns, P. Hostert, T. Krueger, J. Ø. Nielsen, H. Haberl, C. Lauk, J. Lutz and D. Müller (eds.). Land Use Competition: Ecological, Economics and Social Perspectives. Springer, Berlin, Germany.

Gorelick, N., M. Hancher, M. Dixon, S. Ilyushchenko, D. Thau, and R. Moore. 2017. Google Earth Engine: Planetaryscale geospatial analysis for everyone. Remote Sensing of Environment 202:18-27. https://doi.org/10.1016/ j.rse.2017.06.031.

Kutschker, A. M., G. Papazian, O. A. Martínez, and N. Ibañez. 2020. Calidad de los bosques ribereños y perspectivas de restauración en un río de la Patagonia Andina, Argentina. Ecología Austral 30:099-112. https://doi.org/10.25260/ EA.20.30.1.0.928.

Lozano-Báez, S. E., M. Cooper, P. Meli, S. F. B. Ferraz, R. R. Rodrigues, and T. J. Sauer. 2019. Land restoration by tree planting in the tropics and subtropics improves soil infiltration, but some critical gaps still hinder conclusive results. Forest Ecology and Management 444:89-95. https://doi.org/10.1016/j.foreco.2019.04.046.

Macfarlane, W. W., C. M. McGinty, B. G. Laub, and S. J. Gifford. 2016. High-resolution riparian vegetation mapping to prioritize conservation and restoration in an impaired desert river. Restoration Ecology 25(3):333-341. https: //doi.org/10.1111/rec.12425.

Meli, P., A. Calle, Z. Calle, C. I. Ortiz-Arrona, M. Sirombra, and P. H. S. Brancalion. 2019. Riparian-forest buffers: Bridging the gap between top-down and bottom-up restoration approaches in Latin America. Land Use Policy 87: 104085. https://doi.org/10.1016/j.landusepol.2019.104085.

Minneti, J. L. 1999. Atlas climático del Noroeste Argentino. Laboratorio Climatológico sudamericano. Fundación Zon Caldenius, Tucumán, Argentina.

Naiman, R. J., H. Décamps, and M. E. McClain. 2005. Riparia. Ecology, Conservation and Management of Streamside Communities. Elsevier Academic Press. https://doi.org/10.1016/B978-012663315-3/50010-1.

Nanni, A. S., and H. R. Grau. 2017. Land-use redistribution compensated for ecosystem services losses derived from agricultural expansion, with mixed effects on biodiversity in a NW Argentina watershed. Forests 8(8):303. https: //doi.org/10.3390/f8080303.

Olofsson, P., G. M. Foody, S. V. Stehman, and C. E. Woodcock. 2013. Making better use of accuracy data in land change studies: Estimating accuracy and area and quantifying uncertainty using stratified estimation. Remote Sensing of Environment 129:122-131. https://doi.org/10.1016/j.rse.2012.10.031.

ONU (Organización de las Naciones Unidas). 2015. Transforming our world: the 2030 Agenda for sustainable development. A/RES/70/1. URL: tinyurl.com/z4o6pk4.

de Paz, M., M. Gobbi, and E. Raffaele. 2019. Revisión de las experiencias de revegetación con fines de restauración en bosques de la Argentina. Ecología Austral 29:194-207. https://doi.org/10.25260/EA.19.29.2.0.689.

Pérez, D., P. Meli, D. Renison, F. Barri, A. Beider, G. Burgueño, A. Dalmasso, S. Dardanelli, M. de Paz, F. Farinaccio, G. Papazian, M. Sirombra, and R. Torres. 2018. The argentine network of Ecological Restoration (REA): Progress gaps and future pathway. Ecología Austral 28:353-360. https://doi.org/10.25260/EA.18.28.2.0.659.

Pero, E. J. I., and P. Quiroga. 2019. Riparian and adjacent forests differ both in the humid mountainous ecoregion and the semiarid lowland. Plant Ecology 220(4):481-498. https://doi.org/10.1007/s11258-019-00929-w.

Pokrovsky, O. S. 2016. Riparian zones: characteristics, management practices and ecological impacts. Nova Science Publishers, New York, USA.

Pontius Jr., R. G., and M. Millones. 2011. Death to Kappa: birth of quantity disagreement and allocation disagreement for accuracy assessment. International Journal of Remote Sensing 32(15):4407-4429. https://doi.org/10.1080/ 01431161.2011.552923.

Posthumus, H., J. R. Rouquette, J. Morris, D. J. G. Gowing, and T. M. Hess. 2010. A framework for the assessment of ecosystem goods and services; a case study on lowland floodplains in England. Ecological Economics 69:1510-1523. https://doi.org/10.1016/j.ecolecon.2010.02.011. 
Quantum GIS Development Team. 2014. Quantum GIS Geographic Information System. Open Source Geospatial Foundation Project. URL:qgis.osgeo.org.

Riis, T., M. Kelly-Quinn, F. C. Aguiar, P. Manolaki, D. Bruno, M. D. Bejarano, N. Clerici, M. R. Fernandes, J. C. Franco, N. Pettit, A. P. Portela, O. Tammeorg, P. Tammeorg, P. Rodríguez-González, and S. Dufour. 2020. Global Overview of ecosystem services provided by riparian vegetation. BioScience 70(6):501-514. https://doi.org/10.1093/biosci/ biaa041.

Rodríguez-Galiano, V. F., B. Ghimire, J. Rogan, M. Chica-Olmo, and J. P. Rigol-Sánchez. 2012. An assessment of the effectiveness of a random forest classifier for land-cover classification. ISPRS Journal of Photogrammetry and Remote Sensing 67:93-104. https://doi.org/10.1016/j.isprsjprs.2011.11.002.

Roulier, C., C. B. Anderson, S. A. Ballari, and E. A. Nielsen. 2020. Estudios sociales y socio-ecológicos sobre restauración ecológica: Una revisión de la literatura a escala global e iberoamericana. Ecología Austral 30:019-032. https://doi.org/ 10.25260/EA.20.30.1.0.940.

Schweizer, D., P. Meli, P. H. S. Brancalion, and M. R. Guariguata. 2019. Implementing forest landscape restoration in Latin America: Stakeholder perceptions and legal frameworks. Land Use Policy. https://doi.org/10.1016/j.landusep ol.2019.104244.

Sirombra, M. G., and L. M. Mesa. 2012. A method for assessing the ecological quality of riparian forests in subtropical Andean streams: QBRy index. Ecological Indicators 20:324-331. https://doi.org/10.1016/j.ecolind.2012.02.021.

Soria, F. J., C. Fandos, P. Scandaliaris, and J. I. Carreras Baldrés. 2016. Relevamiento satelital de los principales cultivos de Tucumán (2014/2015). Avance Agroindustrial 37(1):1-37. URL: www.eeaoc.gob.ar/?publicacion=av-37-1-9.

Straccia, P. H., and M. L. Isla Raffaele. 2020. Leyes de presupuestos mínimos de protección ambiental. Sobre glaciares, humedales y la emergencia del carácter político de categorías despolitizadas. Ecología Austral 30:085-098. https: //doi.org/10.25260/EA.20.30.1.0.971.

Strassburg, B. B. N., H. L. Beyer, R. Crouzeilles, A. Iribarrem, F. Barros, M. Ferreira de Siqueira, A. Sánchez-Tapia, A. Balmford, J. B. Barreto Sansevero, P. H. S. Brancalion, E. North Broadbent, R. L. Chazdon, A. Oliveira Filho, T. A. Gardner, A. Gordon, A. Latawiec, R. Loyola, J. P. Metzger, M. Mills, H. P. Possingham, R. Ribeiro Rodrigues, C. A. de Mattos Scaramuzza, F. R. Scarano, L. Tambosi, and M. Uriarte. 2019. Strategic approaches to restoring ecosystems can triple conservation gains and halve costs. Nature Ecology and Evolution 3:62-70. https://doi.org/10.1038/s41559018-0743-8.

Suding, K., E. Higgs, M. Palmer, J. Baird Callicott, C. B. Anderson, M. Baker, J. J. Gutrich, K. M. Hondula, M. C. LeFevor, B. M. H. Larson, A. Randall, J. B. Ruhl, and K. Z. S. Schwartz. 2015. Committing to ecological restoration. Science 348(6235):638-640. https://doi.org/10.1126/science.aaa4216.

Tiwari, T., J. Lundström, L. Kuglerová, H. Laudon, K. Öhman, and A. M. Agren. 2016. Cost of riparian buffer zones: A comparison of hydrologically adapted site-specific riparian buffers with traditional fixed widths. Water Resources Research 52:1056-1069. https://doi.org/10.1002/2015WR018014. 